PROCEEDINGS OF THE

AMERICAN MATHEMATICAL SOCIETY

Volume 139, Number 7, July 2011, Pages 2383-2397

S 0002-9939(2010)10677-9

Article electronically published on December 17, 2010

\title{
TOPOLOGICAL CONSTRUCTIONS FOR MULTIGRADED SQUAREFREE MODULES
}

\author{
HARA CHARALAMBOUS
}

(Communicated by Bernd Ulrich)

\begin{abstract}
Let $R=\mathbb{k}\left[x_{1}, \ldots, x_{n}\right]$ and let $M=R^{s} / I$ be a multigraded squarefree module. We discuss the construction of cochain complexes associated to $M$ and we show how to interpret homological invariants of $M$ in terms of topological computations. This is a generalization of the well-studied case of squarefree monomial ideals.
\end{abstract}

\section{INTRODUCTION}

Let $R=\mathbb{k}\left[x_{1}, \ldots, x_{n}\right]$ be a polynomial ring over the field $\mathbb{k}$ of characteristic 0 . For $\alpha=\left(a_{i}\right) \in \mathbb{Z}^{n}$ we let $x^{\alpha}=x_{1}^{a_{1}} \cdots x_{n}^{a_{n}}$ and $R_{\alpha}=k x^{\alpha}$. Let $A=\left(c_{i j} x^{\alpha_{i j}}\right) \in \mathbb{M}_{s \times l}(R)$ : $c_{i j} \in \mathbb{k}$ and $\alpha_{i j} \in \mathbb{N}^{n}$. We say that $A$ is multigraded if each minor of $A$ equals $c_{\alpha} x^{\alpha}$ for some $c_{\alpha} \in \mathbb{k}$. We say that $A$ is of uniform rank if all of its minors are nonzero. In particular this implies that $c_{i j} \neq 0$ for all $i$ and $j$ and thus the matrix of coefficients $\left(c_{i j}\right)$ is sufficiently generic. In addition we say that $\alpha \in \mathbb{N}^{n}$ is squarefree if $\alpha \in\{0,1\}^{n}$ and a collection of vectors is squarefree if each vector is squarefree.

We recall that $M$ is a multigraded $R$-module if $M=\bigoplus_{\alpha \in \mathbb{N}^{n}} M_{\alpha}$, where the $M_{\alpha}$ are subgroups of $M$ and $R_{\alpha_{1}} M_{\alpha_{2}} \subset M_{\alpha_{1}+\alpha_{2}}$ whenever $\alpha_{i} \in \mathbb{N}^{n}$. Moreover $w \in M$ is a multigraded element of $M$ of multidegree $\beta$ if $w \in M_{\beta}$, and in this case we write $\operatorname{deg} w=\beta$. Let $M$ be a multigraded finitely generated $R$-module. $M$ has a minimal multigraded presentation $\phi: R^{l} \longrightarrow R^{s} \longrightarrow M \longrightarrow 0$, where for a choice of multigraded generators for $R^{s}$ and $R^{l}, \phi$ is represented by $A_{M}$, a multigraded presentation matrix of $M$. We note that the data consisting of the multidegrees of the generators of $R^{s}$ and $R^{l}$ and the matrix of coefficients $\left(c_{i j}\right)$ describes a monomial matrix as in MiSt05. In particular whenever $c_{i j} \neq 0, \alpha_{i j}=($ degree of column $j$ )(degree of row $i$ ). We pay special attention to this set of equations. Let $A=$ $\left(c_{i j} x^{\alpha_{i j}}\right) \in \mathbb{M}_{s \times l}(R), c_{i j} \in \mathbb{k}, \alpha_{i j} \in \mathbb{N}^{n}$. Whenever $c_{i j} \neq 0$ we consider the equation $\gamma_{j}-\beta_{i}=\alpha_{i j}$ with unknowns $\gamma_{j}, \beta_{i}$. We assemble these equations to a system $E_{A}$ of at most $s \cdot l$ equations and $s+l$ unknowns. The fact that $A$ is multigraded reflects the consistency of $E_{A}$. For any particular solution $T=\left(\gamma_{1}, \ldots, \gamma_{l}, \beta_{1}, \ldots, \beta_{s}\right)$ of $E_{A}, \gamma_{j}$ gives the degree of the $j^{\text {th }}$ column of $A$ and $\beta_{i}$ the degree of the $i^{\text {th }}$ row of $A$. Moreover for any such solution $T$ we let $F_{1}, F_{0}$ be the free multigraded modules with bases $B_{1}=\left\{w_{j}: j \in[l], \operatorname{deg} w_{j}=\gamma_{j}\right\}$ and $B_{0}=\left\{v_{i}: i \in[s], \operatorname{deg} v_{i}=\beta_{i}\right\}$,

Received by the editors August 28, 2009 and, in revised form, June 30, 2010.

2010 Mathematics Subject Classification. Primary 13C15, 13D02, 13D45.

(C)2010 American Mathematical Society

Reverts to public domain 28 years from publication 
respectively, and let $\phi_{T}: F_{1} \longrightarrow F_{0}, \phi_{T}\left(w_{j}\right)=\sum_{i} c_{i j} x^{\alpha_{i j}} v_{i}$. The module $M_{T}=$ Coker $\phi_{T}$ is multigraded and has $A$ as a presentation matrix. We will occasionally write $M_{A}$ for $M_{T}$.

The multigraded module $M$ is called squarefree if the function $M_{\alpha} \rightarrow M_{\alpha+\beta}$ : $y \mapsto x^{\beta} y$ is a bijection whenever $\operatorname{supp}(\beta) \subset \operatorname{supp}(\alpha)$; see $[$ Ya00]. In some sense it suffices to study squarefree multigraded modules: as is shown in BrHe95 or ChDe01, if $M$ is any multigraded module, then there is a a squarefree multigraded module $L$ with the same homological properties as $M$. In this paper we show that the multigraded matrix $A$ is the presentation matrix of a squarefree module if and only if there exists a squarefree solution to $E_{A}$. Such a matrix is called squarefree. It follows that all nonzero entries of a squarefree matrix have squarefree degrees.

When $M=R / I$ and $I$ is a monomial squarefree ideal, the simplicial complex $\Delta_{I}=\left\{\left\{i_{1}, \ldots, i_{t}\right\} \subset\{1, \ldots, n\}: x_{i_{1}} \cdots x_{i_{t}} \notin I\right\}$, is well studied and properties of $M$ translate to combinatorial properties of $\Delta_{I}$. We generalize the above to any multigraded squarefree module $M$. For this we use a sequence of monomial squarefree ideals that are associated to the presentation of $M$. When the multigraded presentation matrix $A_{M}$ is of uniform rank, such a set of ideals is explicitly computed in terms of the rows of $A_{M}$. A preliminary version of these results (without proofs) has appeared in Ch06.

We describe the main results in each section. We show that if the multigraded squarefree module $M$ has a minimal multigraded presentation $\phi: R^{l} \longrightarrow R^{s} \longrightarrow$ $M \longrightarrow 0$, then there are $s$ squarefree monomial ideals $I_{1}, \ldots, I_{s}$ that determine a multigraded $\mathbb{k}$-basis $M$ (see Theorem 2.3 and Corollary 2.4). This translates as follows in the Gröbner basis language: consider a term order on $R^{s}$ based on any monomial order on $R$ and an ordering of the multigraded basis elements $v_{i}, i \in[s]$ of $R^{s}$; the initial module of the image of $\phi$ is a direct sum $I_{1} v_{1} \oplus \cdots \oplus I_{s} v_{s}$. It follows that if $A$ is any multigraded squarefree matrix, then there is a multigraded squarefree module $M$ with presentation matrix $A$ (see Corollary 2.5). We study the annihilator ideal of a multigraded squarefree module $M_{A}$ when $A$ is an $s \times l$ matrix of uniform rank: when $s>l$ we show that $\operatorname{ann}(M)=0$, while when $s \leq l$ we show that $\operatorname{ann}(M)$ equals the radical of the ideal generated by the $s \times s$ minors of $A$ (see Theorem 2.11).

In the next section we study in more detail the case of a squarefree multigraded module whose presentation matrix $A_{M}$ is of uniform rank. In this case we show that the squarefree monomial ideals that determine a basis of $M$ are generated by least common multiples of monomials in the appropriate rows of $A_{M}$ (see Theorem 3.4). Their intersection equals ann $(M)$. Thus the dimension of $M$ can be computed based on these ideals.

In the last two sections of this paper we assume $M$ to be a squarefree multigraded module. In section 4 , for each $\alpha \in \mathbb{Z}^{n}$ we construct a cochain complex and use it to compute the $\alpha$-graded Betti numbers of $M$. In the last section for each $\alpha \in \mathbb{Z}^{n}$ we construct a complex to calculate the $\alpha$-graded piece of the local cohomology of $M$.

We refer to [Ei97, [BrHe98 and MiSt05] for undefined terms and notation. We also want to thank the referee for suggesting the more general version of Theorem 2.3 and Corollary 2.4 and the generalization of the last two sections. 


\section{Squarefree multigraded matrices}

For $\alpha=\left(a_{i}\right) \in \mathbb{Z}^{n}$, we let $\operatorname{supp}(\alpha)=\left\{i: a_{i} \neq 0\right\} \subset[n]$. When $\alpha \in \mathbb{N}^{n}$ we write $\sigma_{\alpha}$ for $\operatorname{supp}(\alpha)$ and denote by $q_{\alpha}$ the squarefree vector so that $\sigma_{\alpha}=\sigma_{q_{\alpha}}$. If $t \in \mathbb{N}$, by $[t]$ we denote the set $\{1, \ldots, t\}$.

Definition 2.1. A multigraded $s \times l$ matrix $A=\left(c_{i j} x^{\alpha_{i j}}\right)$ is called squarefree if the system $E_{A}$ has a squarefree solution $T$.

Remark 2.2. Let $M$ be a squarefree multigraded module and let $\phi$ be a minimal multigraded presentation $\phi$ of $M$. Since the kernel of $\phi$ is a squarefree module Ya00 it follows that the minimal multigraded generating sets of $M$ and ker $\phi$ have squarefree degrees. Thus $A_{M}$ is squarefree.

Let $T=\left(\gamma_{1}, \ldots, \gamma_{l}, \beta_{1}, \ldots, \beta_{s}\right)$ be a squarefree solution of $E_{A}, \phi_{T}: F_{1} \longrightarrow F_{0}$. For any monomial order on $R$ and an ordering of the basis elements of $F_{0}$, we let $>$ be the following monomial order on $F_{0}: u v_{i}>u^{\prime} v_{j}$ if $v_{i}>v_{j}$ or $i=j$ and $u>u^{\prime}$. We denote by $\operatorname{in}\left(\operatorname{Im} \phi_{T}\right)$ the initial module of $\operatorname{Im}\left(\phi_{T}\right)$ with respect to $>$.

Theorem 2.3. Let $A=\left(c_{i j} x^{\alpha_{i j}}\right)$ be a multigraded squarefree $s \times l$ matrix, $T$ a squarefree solution of $E_{A}$ and $>$ a term order on $F_{0}$ as above. There exist squarefree monomial ideals $I_{1}, \ldots, I_{s}$ of $R$ such that

$$
\operatorname{in}\left(\operatorname{Im} \phi_{T}\right)=I_{1} v_{1} \oplus \cdots \oplus I_{s} v_{s} .
$$

Proof. Without loss of generality we can assume that $v_{s}>\ldots>v_{1}$. Let $f \in \operatorname{Im} \phi$, $f$ multigraded, $\operatorname{deg} f=\alpha, \operatorname{in}(f)=x^{\alpha_{i}} v_{i}$. Thus for $j \in[s]$ and $t \in[l]$, there exist $c_{j} \in \mathbb{k}$ and $r_{t} \in R_{\alpha-\gamma_{t}}$ such that

$$
f=\sum_{j \leq i} c_{j} x^{\alpha_{j}} v_{j}=\sum_{t} r_{t} \phi_{T}\left(w_{t}\right)
$$

For $t \in[l]$, let $d_{t}=\operatorname{deg} r_{t}=\alpha-\gamma_{t}$. Since $\gamma_{t} \in\{0,1\}^{n}$, it follows that whenever $r_{t} \neq 0, d_{t}-\left(\alpha-q_{\alpha}\right)=q_{\alpha}-\gamma_{t} \in\{0,1\}^{n}$. Moreover since $\alpha_{j}=\alpha-\beta_{j}$ and $\beta_{j} \in\{0,1\}^{n}$, it follows that whenever $c_{j} \neq 0, \alpha_{j}{ }^{\prime}=\alpha_{j}-\left(\alpha-q_{\alpha}\right) \in\{0,1\}^{n}$. Thus $r_{t}^{\prime}=r_{t} / x^{\alpha-q_{a}} \in R$ for $t \in[l]$ and

$$
f^{\prime}=\sum_{t} r_{t}{ }^{\prime} \phi_{T}\left(w_{t}\right) \in \operatorname{Im}\left(\phi_{T}\right)
$$

Since $\operatorname{in}\left(f^{\prime}\right)=c_{i} x^{\alpha_{i}{ }^{\prime}} v_{i}$ and $\alpha_{i}-\alpha_{i}{ }^{\prime} \in \mathbb{N}^{n}$ we are done.

We let $M_{T}=$ Coker $\phi_{T}$ and write $\bar{g}$ for $g+\operatorname{Im} \phi_{T}$. We note the following:

Corollary 2.4. Let $A=\left(c_{i j} x^{\alpha_{i j}}\right)$ be a multigraded squarefree $s \times l$ matrix, $T$ a squarefree solution of $E_{A}, M_{T}=$ Coker $\phi_{T}$. There exist simplicial complexes $\Delta_{1}, \ldots, \Delta_{s}$ such that

(1) the set $B\left(M_{T}\right)=\left\{\overline{x^{\beta} v_{i}}: \sigma_{\beta} \in \Delta_{i}, i \in[s]\right\}$ is a $\mathbb{k}$-basis of $M_{T}$;

(2) if $\alpha \in \mathbb{N}^{n}$ and $\sigma(\alpha) \notin \Delta_{i}$, then for each $j<i$ there are unique $r_{i, j, \alpha} \in \mathbb{k}$ such that $r_{i, j, \alpha}=0$ when $\sigma\left(\alpha+\beta_{i}-\beta_{j}\right) \notin \Delta_{j}$ and

$$
\overline{x^{\alpha} v_{i}}=\sum_{j<i} r_{i, j, \alpha} \overline{x^{\alpha+\beta_{i}-\beta_{j}} v_{j}} \text {. }
$$


Proof. We let $I_{1}, \ldots, I_{s}$ be the ideals of Theorem 2.3 with $v_{s}>\ldots>v_{1}$, and we let $\Delta_{i}$ be the simplicial complex $\Delta_{I_{i}}$. The first part follows from Macaulay's Lemma; see for example [Ei97, Theorem 15.3]. For the second part we note that if $\sigma(\alpha) \notin \Delta_{i}$ and $x^{\alpha} \in I_{i}$, then there is an $f_{\alpha} \in \operatorname{Im}\left(\phi_{T}\right)$ such that $\operatorname{in}\left(f_{\alpha}\right)=x^{\alpha} v_{i}$ and

$$
x^{\alpha} v_{i}-f_{\alpha}=\sum_{j<i} c_{j} x^{\alpha_{j}} v_{j}
$$

In particular $\alpha_{j}=\alpha+\beta_{i}-\beta_{j}$. A repeated application of this remark gives the desired result.

The next corollary justifies the definition of a squarefree matrix.

Corollary 2.5. Let $A$ be a multigraded squarefree matrix. Then $A$ is the presentation matrix of a multigraded squarefree module $M$.

Proof. Let $T$ be a squarefree solution of $E_{A}, M_{T}=$ Coker $\phi_{T}$. By Corollary 2.4, $\left(M_{T}\right)_{\alpha} \cong\left(M_{T}\right)_{q_{\alpha}}$ and $M_{T}$ is squarefree.

The join of $\alpha_{1}, \ldots, \alpha_{t} \in \mathbb{N}^{n}$ denoted join $\left(\alpha_{1}, \ldots, \alpha_{t}\right)$ is the vector with components the maximum of the corresponding components of the $\alpha_{1}, \ldots, \alpha_{t}$. We will need the following lemma.

Lemma 2.6. Let $A=\left(c_{i j} x^{\alpha_{i j}}\right)$ be a multigraded $s \times l$ of uniform rank where the $\alpha_{i j}$ are squarefree, and let $t, q \in[l]$ and $f, i_{1}, \ldots, i_{r} \in[s]$. Then

$$
\frac{\operatorname{lcm}\left(x^{\alpha_{t f}}, x^{\alpha_{t i_{1}}}, \ldots, x^{\alpha_{t i_{r}}}\right)}{x^{\alpha_{t f}}}=\frac{\operatorname{lcm}\left(x^{\alpha_{q f}}, x^{\alpha_{q i_{1}}}, \ldots, x^{\alpha_{q i_{r}}}\right)}{x^{\alpha_{q f}}},
$$

or equivalently

$$
\operatorname{join}\left(\alpha_{t f}, \alpha_{t i_{1}}, \ldots, \alpha_{t i_{r}}\right)-\alpha_{t f}=\operatorname{join}\left(\alpha_{q f}, \alpha_{q i_{1}}, \ldots, \alpha_{q i_{r}}\right)-\alpha_{q f} .
$$

Moreover if $f, j_{1}, \ldots, j_{r} \in[s]$ and $q, t \in[l]$, then

$$
\operatorname{join}\left(\alpha_{f t}, \alpha_{j_{1}}, \ldots, \alpha_{j_{r} t}\right)-\alpha_{f t}=\operatorname{join}\left(\alpha_{f q}, \alpha_{j_{1} q}, \ldots, \alpha_{j_{r} q}\right)-\alpha_{f q} .
$$

Proof. We will show the first equality. We note that the last equality is a consequence of the first, since $A^{T}$ is multigraded. The expressions on either side of the equation are squarefree monomials. Suppose that the variable $x_{j}$ divides the left hand side of the expression. This implies that $x_{j}$ does not divide $x^{\alpha_{t f}}$ and $x_{j}$ divides $x^{\alpha_{t i_{h}}}$ for some $i_{h}$, where $h=1, \ldots, r$. Since $c_{t f} c_{q i_{h}} x^{\alpha_{t f}+\alpha_{q i_{h}}}-c_{t i_{h}} c_{q f} x^{\alpha_{t i_{h}}+\alpha_{q f}}$ is a minor of $A$ and $A$ is multigraded, it follows that $x_{j}$ divides $x^{\alpha_{q i_{h}}}$ and $x_{j}$ does not divide $x^{\alpha_{q f}}$. Thus $x_{j}$ divides the right hand side of the equation.

We can now prove the following:

Proposition 2.7. Any multigraded matrix of uniform rank whose entries have squarefree degrees is squarefree.

Proof. Let $A=\left(c_{i j} x^{\alpha_{i j}}\right)$ be a multigraded matrix of uniform rank, where $\alpha_{i j} \in$ $\{0,1\}^{n}$ for $i \in[s], j \in[l]$. By Lemma 2.6] a solution to $E_{A}$ is given by $\gamma_{j}=\operatorname{join}\left(\alpha_{i j}\right.$ : $i \in[s])$ for $j \in[l]$, and $\beta_{i}=\gamma_{1}-\alpha_{i 1}$ for $i \in[s]$.

\section{Remarks 2.8 .}

- We note that when $A$ is of uniform rank then $E_{A}$ has one degree of freedom. 
- When $A=\left(c_{i j} x^{\alpha_{i j}}\right)$ is not of uniform rank then $E_{A}$ might not have squarefree solutions even when $\alpha_{i j} \in\{0,1\}^{n}$ for $c_{i j} \neq 0$. For example, let $R=\mathbb{k}[x, y]$ and

$$
A=\left[\begin{array}{ll}
x & y \\
0 & x
\end{array}\right] .
$$

The general solution of $E_{A}$ consists of $\gamma_{1}=(2+t, s), \gamma_{2}=(1+t, 1+s)$, $\beta_{1}=(1+t, s), \beta_{2}=(t, s+1)$.

- When $A=\left(c_{i j} x^{\alpha_{i j}}\right)$ is of uniform rank and $s=1$ then $B_{0}=\left\{v_{1}: \beta_{1}=\right.$ $\left.\operatorname{deg} v_{1}=0\right\}, B_{1}=\left\{w_{j}: \gamma_{j}=\operatorname{deg} w_{j}=\alpha_{1 j}\right\}$ and $M_{T}=R / I$, where $I=\left\langle x^{a_{11}}, \ldots, x^{a_{1 l}}\right\rangle$.

We will close this section by examining the annihilator of a multigraded squarefree module $M$ in terms of its multigraded presentation matrix $A$. It is easy to see that $\operatorname{ann}(M)$ is generated by monomials. If $A$ is $s \times l$ and $l \geq s$ we denote by $\operatorname{Fitt}_{0}(M)$ the ideal of the $s \times s$ minors of $A$. We note that the generators of $\sqrt{\text { Fitt }_{0}(M)}$ are least common multiples of the entries in a diagonal of $A$ of length $s$. It is well known (see [Ei97]) that $\operatorname{Fitt}_{0}(M) \subset \operatorname{ann}(M)$ and that $\operatorname{ann}(M)^{s} \subset \operatorname{Fitt}_{0}(M)$. In the case where $\operatorname{ann}(M)$ is a monomial ideal it follows that $\operatorname{ann}(M) \subseteq \sqrt{\operatorname{Fitt}_{0}(M)}$. For what follows we write $\operatorname{diag}\left(x^{\alpha}, s\right)$ for the $s \times s$ identity matrix times $x^{\alpha}$. We will use the following lemma, which characterizes the elements of $\operatorname{ann}(M)$.

Lemma 2.9. Let $A$ be multigraded squarefree $s \times l$ matrix and let $M$ be a module with presentation matrix $A$. The annihilator of $M$ consists of all monomials $x^{\alpha}$ such that the linear system

$$
A X=\operatorname{diag}\left(x^{\alpha}, s\right)
$$

has a solution in $\mathbb{M}_{l \times s}(R)$.

Proof. Let $\phi: F_{1} \longrightarrow F_{0} \longrightarrow M \longrightarrow 0$ be such that for a basis $\left\{v_{i}: i \in[s]\right\}$ of $F_{0}$ and a basis $\left\{w_{j}: j \in[l]\right\}$ of $F_{1}, \phi$ is represented by $A=\left(c_{i j} x^{\alpha_{i j}}\right)$. Since $M=F_{0} / \operatorname{Im}(\phi)$, it follows that $x^{\alpha} \in \operatorname{ann}(M)$ if and only if $x^{\alpha} v_{i} \in \operatorname{Im}(\phi)$. Thus $x^{\alpha} \in \operatorname{ann}(M)$ if and only if for any $i \in[s]$ there exist $r_{i 1}, \ldots, r_{i l}$ such that

$$
x^{\alpha} v_{i}=\sum_{j=1}^{l} r_{i j} \sum_{t=1}^{s}\left(c_{t j} x_{t j}^{\alpha}\right) v_{t} .
$$

We let $C_{i}$ be the $i$ th column of $\operatorname{diag}\left(x^{\alpha}, s\right)$. Since $\left\{v_{i}: i \in[s]\right\}$ is a basis for $F_{0}$, the system (2.10) is consistent if and only if $A X=C_{i}$ is consistent for each $i \in[s]$.

If $K_{1} \subset[s]$ and $K_{2} \subset[l]$ we denote by $A\left[K_{2}, K_{1}\right]$ the submatrix of $A$ consisting of the rows indexed by $K_{1}$ and the columns indexed by $K_{2}$. The next proposition computes the annihilator of the squarefree module $M$ when the presentation matrix is of uniform rank.

Proposition 2.11. Let $A$ be a multigraded squarefree $s \times l$ matrix of uniform rank. Suppose that $A$ is the presentation matrix of $M$. If $l<s$, then $\operatorname{ann}(M)=0$. Otherwise $\operatorname{ann}(M)=\sqrt{\text { Fitt }_{0}(M)}$.

Proof. If $l<s$ and $x^{\alpha} \in \operatorname{ann}(M)$, then by Lemma 2.9 assume that $Z$ is such that

$$
A Z=\operatorname{diag}\left(x^{\alpha}, s\right) .
$$


Without loss of generality we can assume that the first column $Z_{1}$ of $Z$ is nonzero. It follows that $A[[l],\{2, \ldots, l+1\}] \cdot Z_{1}=0$ and thus $\operatorname{det} A[[l],\{2, \ldots, l+1\}]=0$, a contradiction since $A$ is of uniform rank.

Suppose now that $l \geq s$. We will show that $\sqrt{\operatorname{Fitt}_{0}(M)} \subset \operatorname{ann}(M)$. Let $I=$ $\sqrt{\text { Fitt }_{0}(M)}$ and let $x^{\alpha}$ be a minimal generator of $I$. It follows that there is a set $K=\left\{j_{1}, \ldots, j_{s}: j_{1}<j_{2}<\ldots<j_{s}\right\} \subset[l]$ such that $x^{\alpha}=\operatorname{det} A[K,[s]]$. Since $A$ is multigraded, it follows that for any $i \in[s], \operatorname{det} A[K,[s]]=c_{K, t} x^{\alpha_{i j_{t}}} \operatorname{det} A[K \backslash$ $\left.j_{t},\{1, \ldots, \hat{i}, \ldots, s\}\right]$, where $c_{K, t} \in \mathbb{k}$. For $i \in[s]$, we let $C_{i}$ be as in the proof of Lemma 2.9. By Cramer's rule it follows that the system $A[K,[s]] X=C_{i}$ has a solution $Z=\left(z_{j 1}\right) \in \mathbb{M}_{s \times 1}(R)$ with entries in $R$. We extend $Z$ to a solution $Y \in \mathbb{M}_{l \times 1}(R)$ for the system $A X=C_{i}$ by setting $y_{j 1}=z_{j 1}$ when $j \in K$ and letting $y_{j 1}=0$ if $j \notin K$. Thus by Lemma 2.9, $x^{\alpha} \in \operatorname{ann}(M)$ and $I \subset \operatorname{ann}(M)$, as desired.

\section{Squarefree matrices of UNiform RANK}

In the previous section for any multigraded squarefree matrix $A$ and any solution $T$ of $E_{A}$ we proved the existence of a sequence of squarefree monomial ideals that provide a basis for $M_{T}$. In this section we compute these ideals when $A$ is squarefree of uniform rank.

Definition 3.1. Let $A=\left(c_{i j} x^{\alpha_{i j}}\right)$ be a multigraded squarefree matrix of uniform rank with $l \geq s$. For $i \in[s]$ we let

$$
I_{i}:=\left\langle\operatorname{lcm}\left(x^{\alpha_{i j_{1}}}, \ldots, x^{\alpha_{i j_{s-i+1}}}\right): 1 \leq j_{1}<\cdots<j_{s-i+1} \leq l\right\rangle .
$$

We denote by $\Delta_{i}$ the simplicial complex $\Delta_{I_{i}}$.

For the rest of this section we assume that $A$ is of uniform rank as above.

Example 3.2. Let $R=\mathbb{k}[x, y, z, w]$ and

$$
A=\left[\begin{array}{cc}
x y & x z \\
w y & 2 w z
\end{array}\right] .
$$

$A$ is the matrix of the $R$-module homomorphism $\phi_{1}: F_{1} \longrightarrow F_{0}$, where $F_{1}=R w_{1} \oplus$ $R w_{2}, F_{0}=R v_{1} \oplus R v_{2}, \operatorname{deg} w_{1}=\gamma_{1}=(1,1,0,1), \operatorname{deg} w_{2}=\gamma_{2}=(1,0,1,1), \operatorname{deg} v_{1}=$ $\beta_{1}=(0,0,0,1), \operatorname{deg} v_{2}=\beta_{2}=(1,0,0,0), M_{A}=\operatorname{Coker} \phi_{1}$. Here $I_{1}=(x y z)$ and $I_{2}=(w y, w z)$. Below we graph the simplicial complexes $\Delta_{1}, \Delta_{2}$.
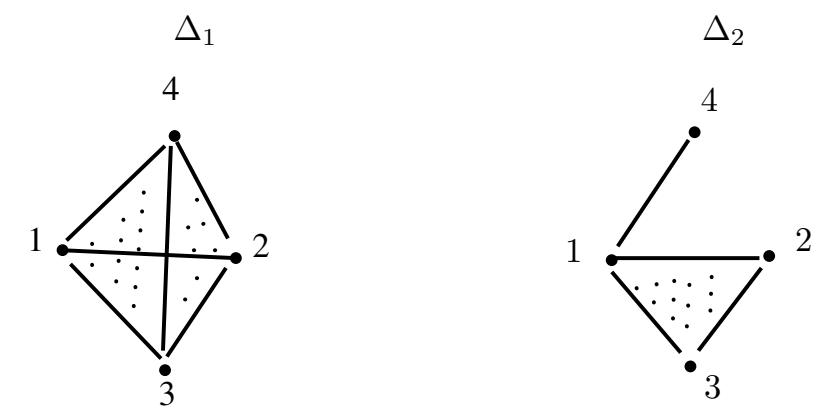

We note that when $s=1$, then $I_{1}=\left\langle x^{a_{11}}, \ldots, x^{a_{1 l}}\right\rangle$ and the unique multigraded $\mathbb{k}$-basis of $R / I_{1}$ is the set $\left\{x^{\beta}: \sigma_{\beta} \notin \Delta_{1}\right\}$. We recall that if $T=\left(\gamma_{j}: j \in[l], \beta_{i}: i \in\right.$ $[s])$ is a solution of $E_{A}$, then $F_{1}, F_{0}$ are the free multigraded modules with bases 
$B_{1}=\left\{w_{j}: j \in[l], \operatorname{deg} w_{j}=\gamma_{j}\right\}, B_{0}=\left\{v_{i}: i \in[s], \operatorname{deg} v_{i}=\beta_{i}\right\}$, respectively, $\phi_{T}: F_{1} \longrightarrow F_{0}, \phi_{T}\left(w_{j}\right)=\sum_{i} c_{i j} x^{\alpha_{i j}} v_{i}$ and $M_{A}=$ Coker $\phi_{T}$.

Definition 3.3. We define

$$
\begin{aligned}
\mathcal{B}_{j A}:=\left\{\overline{x^{\beta} v_{j}}:\right. & \left.x^{\beta} \notin I_{j}\right\}=\left\{\overline{x^{\beta} v_{j}}: \sigma_{\beta} \in \Delta_{j}\right\}, \\
\mathcal{B}_{A}: & =\bigcup_{j=1}^{s} \mathcal{B}_{j A} .
\end{aligned}
$$

The elements of $\mathcal{B}_{A}$ are multigraded and $\operatorname{deg} \overline{x^{\beta} v_{j}}=\beta+\beta_{j}$.

Theorem 3.4. Let $A=\left(c_{i j} x^{\alpha_{i j}}\right)$ be an $s \times l$ multigraded squarefree matrix of uniform rank. The set $\mathcal{B}_{A}$ is a multigraded $\mathbb{k}$-basis for $M_{A}$.

Proof. We use induction on $s$. For $s=1$ the theorem is clear. Let $s>1$. First we show that the elements of $\mathcal{B}_{A}$ are linearly independent. Suppose that

$$
\sum_{j \in[s]} \sum_{i \in K_{j}} k_{j i} x^{\beta_{j i}} v_{j}=\sum_{f=1}^{l} \sum_{t=1}^{s} r_{f}\left(c_{t f} x^{\alpha_{t f}}\right) v_{t}
$$

is a dependence relation; $K_{j}$ is a finite index set for each $j \in[s], k_{j i} \in \mathbb{k}$, and $\overline{x^{\beta_{j i}} v_{j}} \in \mathcal{B}_{j A}$. It follows that

$$
\sum_{i \in K_{1}} k_{1 i} x^{\beta_{1 i}} v_{1}=\sum_{f=1}^{l} r_{f} c_{1 f} x^{\alpha_{1 f}} v_{1}
$$

while

$$
\sum_{t=2}^{s} \sum_{i \in K_{t}} k_{t i} x^{\beta_{j i}} v_{t}=\sum_{f=1}^{l} \sum_{t=2}^{s} r_{f} c_{t f} x^{\alpha_{t f}} v_{t} .
$$

Let $F_{0}{ }^{\prime}=R v_{2} \oplus \cdots \oplus R v_{s}, \phi^{\prime}: F_{1} \longrightarrow F_{0}{ }^{\prime}$ be the $R$-homomorphism whose matrix with respect to the bases $\left\{w_{i}: i \in[l]\right\}$ of $F_{1}$ and $\left\{v_{i}: i \in\{2, \ldots, s\}\right\}$ of $F_{0}{ }^{\prime}$ is the submatrix $A^{\prime}=A[\{1, \ldots, l\},\{2, \ldots, s\}]$ of $A$. Thus $M_{A^{\prime}}=F_{0}{ }^{\prime} / \operatorname{Im} \phi^{\prime}$. We note that for $j=1, \ldots, s-1$ there is a one-to-one correspondence between the elements of $\mathcal{B}_{j A^{\prime}}$ and $\mathcal{B}_{j+1 A}$. Thus the expression of equation (3.6) translates to a dependence relation for the elements $\mathcal{B}_{A^{\prime}}$. According to the induction hypothesis this implies that $k_{t i}=0$ for $t \geq 2$ and $i \in K_{t}$. Thus it remains to show that $k_{1 i}=0$. We examine the coefficients $r_{f}$ that appear on the right hand side of equations (3.5) and (3.6). Since the sum on the left hand side of equation (3.6) is zero, it follows that $r_{1} w_{1}+\cdots+r_{l} w_{l} \in \operatorname{ker} \phi_{1}^{\prime}$. According to the description of the free resolution of $M_{A^{\prime}}$ (see [ChTc03]) it follows that for $1 \leq j \leq l$,

$$
r_{j} \in\left\langle\frac{\operatorname{lcm}\left(x^{\alpha_{2 j}}, x^{\alpha_{2 i_{1}}}, \cdots, x^{\alpha_{2 i_{s-1}}}\right)}{x^{\alpha_{2 j}}}: 1 \leq i_{1}<\cdots<i_{s-1} \leq l, i_{t} \neq j\right\rangle .
$$

By Lemma 2.6.

$$
\frac{\operatorname{lcm}\left(x^{\alpha_{2 j}}, x^{\alpha_{2 i_{1}}}, \cdots, x^{\alpha_{2 i_{s-1}}}\right)}{x^{\alpha_{2 j}}}=\frac{\operatorname{lcm}\left(x^{\alpha_{1 j}}, x^{\alpha_{1 i_{1}}}, \cdots, x^{\alpha_{1 i_{s-1}}}\right)}{x^{\alpha_{1 j}}}
$$

and $r_{j} x^{\alpha_{1 j}} \in I_{1}$, a contradiction.

Next we show that $\mathcal{B}_{A}$ generates $M_{A}$. More precisely we will show that if $x^{\alpha} \in I_{i}$, then $\overline{x^{\alpha} v_{i}}$ can be written as a $\mathbb{k}$-linear combination of elements of $\mathcal{B}_{1 A} \cup \ldots \cup \mathcal{B}_{i-1 A}$ of degree $\alpha+\beta_{i}$. We first show this for the elements of $I_{1}$. Let $x^{\alpha}$ be the least common 
multiple of the entries in the first row corresponding to the columns indexed by the set $K \subset[l]$, where $|K|=s$. Since $A$ is multigraded, $\operatorname{det} A[K,[s]]$ divides $x^{\alpha} \operatorname{det} A[K \backslash f,\{2, \ldots, s\}]$ for any $f \in K$. By Cramer's rule it follows that there is a matrix $Z=\left(z_{i}\right) \in \mathbb{M}_{s \times 1}(R)$ such that

$$
A[K,[s]] Z=\left[\begin{array}{llll}
x^{\alpha} & 0 & \cdots & 0
\end{array}\right]^{T} .
$$

It follows that if $K=\left\{i_{1}, \ldots, i_{s}\right\}$, then $x^{\alpha} v_{1}=\phi_{1}\left(z_{1} w_{i_{1}}+\cdots+z_{s} w_{i_{s}}\right)$. Therefore $\overline{x^{\alpha} v_{1}}=0$. We now assume that the statement holds for $j<t$. Let $K=$ $\left\{i_{1}, \ldots, i_{s-t+1}\right\}$ and $x^{\alpha} \in I_{t}$ be equal to $\operatorname{lcm}\left(x^{a_{t i_{f}}}: i_{f} \in K\right)$. Let $Z=\left(z_{f}\right) \in$ $\mathbb{M}_{(s-t+1) \times 1}(R)$ be such that

$$
A[K,\{t, \ldots, s\}] Z=\left[\begin{array}{llll}
x^{\alpha} & 0 & \cdots & 0
\end{array}\right]^{T} .
$$

For $w=z_{1} w_{i_{1}}+\cdots+z_{s-t+1} w_{i_{s-t+1}}$ we have:

$$
\begin{aligned}
\phi_{1}(w) & =z_{1}\left(\sum_{j<t} c_{j i_{1}} x^{\alpha_{j i_{1}}} v_{j}\right)+\cdots+z_{s-t+1}\left(\sum_{j<t} c_{j i_{s-t+1}} x^{\alpha_{j i_{s-t+1}}} v_{j}\right)+x^{\alpha} v_{t} \\
& =\sum_{j<t}\left(\sum_{r=1}^{r=t+1} z_{r} c_{j i_{r}} x^{\alpha_{j i_{r}}} v_{j}\right)+x^{\alpha} v_{t}
\end{aligned}
$$

Therefore

$$
\overline{x^{\alpha} v_{t}}=-\sum_{j<t}\left(\sum_{r=1}^{r=s-t+1} c_{j i_{r}} \overline{z_{r} x^{\alpha_{j i_{r}} v_{j}}}\right),
$$

and we are done by the induction hypothesis as applied to each of the summands $\overline{z_{r} x^{\alpha_{j i r}} v_{j}}$.

The next corollary is immediate, and we omit its proof.

Corollary 3.7. Let $A$ be an $s \times l$ multigraded squarefree matrix of uniform rank, and let $>$ be a term order on $F_{0}$ based on a monomial order of $R$ with $v_{s}>\cdots>v_{1}$. Then

$$
\operatorname{in}\left(\operatorname{Im}\left(\phi_{1}\right)\right)=I_{1} v_{1} \oplus \cdots \oplus I_{s} v_{s} .
$$

Example 3.8. Let $A$ be the matrix of Example 3.2 and $\alpha=(0,1,0,1)$. Then $\overline{w y v_{2}}=-\overline{x y v_{1}}$ and $r_{2,1, \alpha}=-1$. When $\alpha=(0,0,1,1)$ then $\overline{w z v_{2}}=-1 / 2 \overline{x z v_{1}}$ and $r_{2,1, \alpha}=-1 / 2$. Note that $x y z v_{1}=\phi_{1}\left(2 z w_{1}-y w_{2}\right)$ and $\overline{x y z v_{1}}=0$.

For a different ordering of the basis elements $v_{i}$, one would modify Definition 3.1 to get a different set of ideals and a potentially different $\mathbb{k}$-multigraded basis of $M_{A}$. For example if $v_{1}>\cdots>v_{s}$, then the $i^{\text {th }}$ ideal should be generated by all least common multiples of $i$ monomials in the $i^{\text {th }}$ row of $A$. Next we describe the annihilator of $M$ in terms of the ideals $I_{i}$.

Proposition 3.9. Let $A=\left(c_{i j} x^{\alpha_{i j}}\right)$ be an $s \times l$ multigraded squarefree matrix of uniform rank, $l \geq s$. Then

$$
\operatorname{ann}\left(M_{A}\right)=I_{1} \cap \cdots \cap I_{s}
$$

Proof. We use Proposition 2.11, We first show that the intersection $I_{1} \cap \cdots \cap I_{s}$ is contained in $\operatorname{ann}\left(M_{A}\right)$. Let $x^{\alpha_{i}} \in I_{i}$ for each $i \in[s]: x^{\alpha_{i}}$ determines a (not necessarily unique) subset $L_{i} \subset[l]$ of cardinality $s-i+1$ such that $x^{\alpha_{i}}=\operatorname{lcm}\left(x^{\alpha_{i t}}\right.$ : $\left.t \in L_{i}\right)$. It follows that there is a set $K=\left\{i_{1}, \ldots, i_{s}\right\} \subset[l]$ of cardinality $s$ such that $i_{t} \in L_{t}$. Since $A$ is multigraded it follows that $\operatorname{det} A[K,[s]] \operatorname{divides} \operatorname{lcm}\left(\alpha_{1}, \ldots, \alpha_{s}\right)$. 
For the reverse containment, let $x^{q}$ be a generator of $\operatorname{ann}\left(M_{A}\right)$ : thus there is an ordered set $K=\left\{j_{1}, \ldots, j_{s}\right\}$ such that $c x^{\alpha}=\operatorname{det} A[K,[s]]$ and $q=q_{\alpha}$. Let $x^{\alpha_{i}}=\operatorname{lcm}\left(x^{\alpha_{i j_{t}}}: t=i, \ldots, s\right)$. It is clear that $x^{\alpha_{i}} \in I_{i}$. Since $A$ is multigraded it follows that $x^{q_{\alpha}}=\operatorname{lcm}\left(x^{\alpha_{1}}, \ldots, x^{\alpha_{s}}\right)$.

The following is now immediate:

Corollary 3.10. Let $A$ be as above. The dimension of $M_{A}$ is equal to the least of the codimensions of the ideals $I_{j}$.

\section{Betti numbers of $M$}

Let $A=\left(c_{i j} x^{\alpha_{i j}}\right)$ be an $s \times l$ multigraded squarefree matrix, $T$ a squarefree solution of $E_{A}, M_{A}=$ Coker $\phi_{T}$. We consider the ideals $I_{1}, \ldots, I_{s}$ of Theorem 2.3 with respect to a term order induced by $v_{s}>\cdots>v_{1}$. We start by assembling some notation.

\section{Notation 4.1.}

- For $i \in[s]$, we let $\beta_{i}=\operatorname{deg}\left(v_{i}\right)$. For $\alpha \in \mathbb{Z}^{n}$ we let $\alpha_{j}=\alpha-\beta_{j}$.

- Let $L \subset[n]$. If $t \in L$, then we write $L \hat{t}$ for the set $L \backslash\{t\}$. If $\sigma \subset L$ we write $L \hat{\sigma}$ for the set $L \backslash \sigma$.

- By $L t$ we denote the set $L \cup\{t\}$; by $L \sigma$ we denote the set $L \cup \sigma$.

- Let $L \subset[n]$. Then $\underline{L}=\left(d_{i}\right)$, where $d_{i}=1$ if $i \in L$ and 0 otherwise.

- Let $L=\left\{i_{1}, \ldots, i_{t}\right\}$, where $1 \leq i_{1}<\ldots<i_{t} \leq n$. For $r \in[t]$ we let $\operatorname{sgn}\left[i_{r}, L\right]=(-1)^{r+1}$. For $W \subset L$ we let

$$
\operatorname{sgn}[W, L]:=\prod_{w \in W} \operatorname{sgn}[w, L] .
$$

- Let $\left(K_{\bullet}, \theta_{\bullet}\right)$ be the Koszul complex on the variables $x_{1}, \ldots, x_{n}$. We denote the multigraded generators of $K_{j}$ by $e_{L}$ where $L=\left\{i_{1}, \ldots, i_{j}\right\}$ and $1 \leq$ $i_{1}<\ldots<i_{j} \leq n$ and let $\operatorname{deg} e_{L}=\underline{L}$. Here

$$
\theta\left(e_{L}\right)=\sum_{t \in L} \operatorname{sgn}[t, L] x_{t} e_{L \hat{t}} .
$$

- Let $\Delta$ be a simplicial complex, $\tau \in \Delta$ and $V$ the vertex set of $\Delta$. We partition $V \backslash \tau$ into two sets: $V_{\tau, \Delta, 1}=\{t \notin \tau: \tau t \in \Delta\}\left(=\operatorname{link}_{\Delta} \tau\right)$ and $V_{\tau, \Delta, 2}=\{t \notin \tau: \tau t \notin \Delta\}$.

- Let $\Delta$ be a simplicial complex. We let $C^{j}(\Delta)$ be the $\mathbb{k}$-vector space with basis elements $\tau^{*}$, where $\tau \in \Delta$ and $|\tau|=j+1$. We let $\left(C^{\bullet}(\Delta), d\right)$ be the augmented cochain complex

$$
C^{\bullet}(\Delta): \quad 0 \longrightarrow C^{-1}(\Delta) \longrightarrow C^{0}(\Delta) \stackrel{d^{0}}{\longrightarrow} \cdots \longrightarrow C^{n-1}(\Delta) \longrightarrow 0,
$$

where

$$
d^{j}\left(\tau^{*}\right)=\sum_{t \in V_{\tau, \Delta, 1}} \operatorname{sgn}[t, \tau t](\tau t)^{*} .
$$

We let $\widetilde{H}^{i}(\Delta)=H^{i}\left(C^{\bullet}(\Delta)\right)$.

- For $\alpha \in \mathbb{Z}^{n}$, we write $\alpha=\alpha^{+}-\alpha^{-}$, where $\alpha^{+}, \alpha^{-} \in \mathbb{N}^{n}$ and $\operatorname{supp}\left(\alpha^{+}\right) \cap$ $\operatorname{supp}\left(\alpha^{-}\right)=\emptyset$.

- Let $\Delta$ be a simplicial complex and $\alpha \in \mathbb{N}^{n}$. We let

$$
\Delta_{\alpha}=\left\{\sigma \subset \sigma_{\alpha}: \sigma \cup \sigma_{\alpha-q_{\alpha}} \in \Delta\right\} .
$$

If $\alpha \in \mathbb{Z}^{n} \backslash \mathbb{N}^{n}$ we let $\Delta_{\alpha}=\{\}$. 
- We let $\Delta_{j, \alpha}(A)$ or $\Delta_{j, \alpha}$ for short be the simplicial complex $\left(\Delta_{I_{j}}\right)_{\alpha_{j}}$. Thus

$$
\Delta_{j, \alpha}(A)=\left\{\sigma \subset \sigma_{\alpha_{j}}: \sigma \cup \sigma_{\alpha_{j}-q_{\alpha_{j}}} \in \Delta_{I_{j}}\right\} .
$$

- We let

$$
\left(C^{\bullet}(j, \alpha), d_{j}\right)=\left(C^{\bullet}\left(\Delta_{j, \alpha}\right), d_{j}\right) .
$$

- Let $\tau \subset[n]$ be such that $\sigma_{\beta_{i}} \subset \tau \sigma_{\beta_{j}}$. We define $f(\tau, j, i)=\sigma_{\beta_{j}} \tau \widehat{\sigma_{\beta_{i}}}$.

- Let $\tau^{*} \in C^{r}(j, \alpha)$. Let $w \in[n]$ be such that $\tau w \notin \Delta_{j, \alpha}$. The coefficient $r_{j, i, \underline{w} \tau+\alpha_{j}-q_{\alpha_{j}}}$ is determined by Corollary 2.4. Whenever $r_{j, i, \underline{w \tau}+\alpha_{j}-q_{\alpha_{j}}} \neq 0$ it follows that $\sigma_{\beta_{i}} \subset \tau \sigma_{\beta_{j}}$ and $f(\tau, j, i) \in \Delta_{i, \alpha}$ so that

$$
\chi_{j}\left(\tau^{*}, w\right)=\operatorname{sgn}[w, \tau w] r_{j, i, \underline{w \tau}+\alpha_{j}-q_{\alpha_{j}}} \frac{\operatorname{sgn}\left[\tau w, \sigma_{\alpha_{j}}\right]}{\operatorname{sgn}\left[f(\tau w, j, i), \sigma_{\alpha_{i}}\right]}(f(\tau w, j, i))^{*}
$$

is an element of

$$
\sum_{i<j} C^{r+1+\left(\left|\sigma_{\alpha_{i}}\right|-\left|\sigma_{a_{j}}\right|\right)}(i, \alpha)
$$

We let

$$
\chi_{j}\left(\tau^{*}\right)=\sum_{w \in V_{\tau, \Delta_{j, \alpha}, 2}} \chi_{j}\left(\tau^{*}, w\right) .
$$

Example 4.2. Let $A$ be the matrix of Example 3.2. Let $\alpha=(1,0,1,1)$. Then $\alpha_{1}=(1,0,1,0), \alpha_{2}=(0,0,1,1), \sigma_{\alpha_{1}}=\{1,3\}, \sigma_{\alpha_{2}}=\{3,4\}$ while $\sigma_{\beta_{1}}=\{4\}$ and $\sigma_{\beta_{2}}=\{1\} . \quad \Delta_{1, \alpha}$ is the line segment between the vertices 1 and 3 , while $\Delta_{2, \alpha}$ consists of the points 3 and 4 . It follows that $f(\{3,4\}, 2,1)=\{1,3\}$, an element of $\Delta_{1, \alpha}$ and $r_{2,1,\{3,4\}}=-1 / 2$. Thus $\chi_{2}\left(\{3\}^{*}\right)=-\frac{1}{2}\{1,3\}^{*}$.

Next we turn our attention to the minimal multigraded free resolutions of $M_{A}$. Let $\alpha \in \mathbb{Z}^{n}$ and $b_{i, \alpha}\left(M_{A}\right)$ be the $\alpha$-graded $i$-Betti number of $M_{A}$ :

$$
b_{i, \alpha}\left(M_{A}\right)=\operatorname{dim}_{\mathbb{k}} \operatorname{Tor}_{i}\left(M_{A}, k\right)_{\alpha}=\operatorname{dim}_{\mathbb{k}} H_{i}\left(M_{A} \otimes K_{\bullet}\right)_{\alpha}=\operatorname{dim}_{\mathbb{k}} F_{i} \otimes \mathbb{k},
$$

where $F_{\bullet}: \quad 0 \longrightarrow F_{p} \longrightarrow \cdots \cdots \longrightarrow F_{1} \stackrel{\phi_{1}}{\longrightarrow} F_{0} \longrightarrow M_{A} \longrightarrow 0$ is a minimal multigraded free resolution of $M_{A}\left(\phi_{1}=\phi_{T}\right)$. It is well known that when $I$ is a squarefree ideal then $b_{i, \alpha}(R / I)=\widetilde{H}^{\left|\sigma_{\alpha}\right|-i-1}\left(C^{\bullet}\left(\left(\Delta_{I}\right)_{\alpha}\right)\right)$; see [Ho77] or [MiSt05] for a proof. More precisely there is an isomorphism of complexes,

$$
\left(R / I \otimes K_{\bullet}\right)_{\alpha} \cong C^{\bullet}\left(\left(\Delta_{I}\right)_{\alpha}\right)
$$

such that

$$
\left(R / I \otimes K_{i}\right)_{\alpha} \cong C^{\left|\sigma_{\alpha}\right|-i-1}\left(\left(\Delta_{I}\right)_{\alpha}\right) .
$$

We generalize the isomorphism (4.3) for $M_{A}$. We will need the following remark on the signs, proved essentially in $\mathrm{Ho} 77$.

Remark 4.4. Let $\rho \subset \sigma, \tau=\sigma \backslash \rho$ and $t \in \tau$. Then

$$
\operatorname{sgn}[t, \tau] \operatorname{sgn}[\rho t, \sigma]=\operatorname{sgn}[t, \rho t] \operatorname{sgn}[\rho, \sigma] .
$$

We combine the cochain complexes of $\Delta_{j, \alpha}$ to construct a new complex.

Construction 4.5. Let $l_{j}=\left|\sigma_{\alpha_{j}}\right|-\left|\sigma_{\alpha_{1}}\right|$. We define

$$
C^{t}(A, \alpha):=\sum_{j=1}^{s} C^{t+l_{j}}(j, \alpha),
$$


and let $\delta^{t}: C^{t}(A, \alpha) \longrightarrow C^{t+1}(A, \alpha)$ be such that for $\tau^{*} \in C^{t+l_{j}}(j, \alpha)$,

$$
\delta^{t}\left(\tau^{*}\right):=d_{j}^{t}\left(\tau^{*}\right)+\chi_{j}\left(\tau^{*}\right) .
$$

Theorem 4.6. Let $A$ be a squarefree multigraded matrix. $\left(C^{\bullet}(A, \alpha), \delta^{\bullet}\right)$ is a cochain complex and there is an isomorphism of complexes $\left(C^{\bullet}(A, \alpha), \delta^{\bullet}\right) \cong(A \otimes$ $\left.K_{\bullet}\right)_{\alpha}$.

Proof. First we note that $M_{A} \otimes K_{\bullet}$ is multigraded. By Corollary 2.4, a multigraded basis for the vector space $\left(M_{A} \otimes K_{\bullet}\right)_{\alpha}$ is

$$
\begin{aligned}
& \bigcup_{j} \bigcup_{\substack{L \subset[n] \\
\alpha_{j}-\operatorname{deg} L \in \mathbb{N}^{n}}}\left\{\overline{x^{\alpha_{j}-\operatorname{deg} L} v_{j}} \otimes e_{L}: \overline{x^{\alpha_{j}-\operatorname{deg} L} v_{j}} \in B\left(M_{A}\right)\right\} \\
& =\bigcup_{\substack{L \\
\alpha_{j}-\operatorname{deg} L \in \mathbb{N}^{n}}} \bigcup_{j}\left\{\overline{x^{\alpha_{j}-q_{\alpha_{j}}} x^{q_{\alpha_{j}}-\operatorname{deg} L} v_{j}} \otimes e_{L}: \sigma_{\alpha_{j}} \hat{L} \in \Delta_{j, \alpha}\right\} .
\end{aligned}
$$

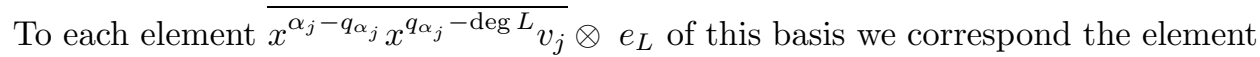
$\operatorname{sgn}\left[\sigma_{\alpha_{i}} \backslash L, \sigma_{\alpha_{i}}\right]\left(\sigma_{\alpha_{i}} \backslash L\right)^{*}$ of $C^{r}(j, \alpha)$, where $r=\left|\sigma_{\alpha_{j}}\right|-|L|-1$. Since $\left(M_{A} \otimes K_{\bullet}\right)_{\alpha}$ is a complex, to prove our claim it suffices to show that the following diagram commutes:

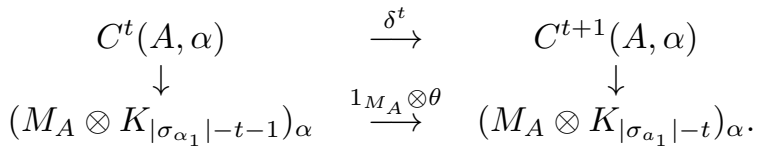

This is a routine check, using Remark 4.4 .

The following is now immediate and generalizes the well-known formula of the cyclic case.

Corollary 4.7. Let $A$ be as above. Then

$$
b_{i, \alpha}\left(M_{A}\right)=H^{\left|\sigma_{\alpha_{1}}\right|-i-1}\left(C^{\bullet}(A, \alpha)\right) .
$$

When $E^{\bullet}$ is a complex, by $E^{\bullet}[-1]$ we mean the complex $E^{\bullet}$ pushed in homological degree $-1: E^{r}[-1]:=C^{r-1}$. This way we can think of $C^{\bullet}(A, \alpha)$ as the cochain complex that results by a succession of mapping cones $\mathbb{M}\left(f_{i}\right)$. We start with $\mathbb{M}\left(f_{1}\right)=C^{\bullet}(1, \alpha)[-1]$, and once $\mathbb{M}\left(f_{i-1}\right)$ has been constructed, then $\mathbb{M}\left(f_{i}\right)$ is the cokernel of

$$
f_{i}:\left(C^{\bullet}(i, \alpha)\left[-\left|\sigma_{\alpha_{i}}\right|+\left|\sigma_{a_{1}}\right|\right],-d\right) \stackrel{d_{i}^{\prime}}{\longrightarrow} \mathbb{M}\left(f_{i-1}\right)[-1] .
$$

We note that if $\alpha \in \mathbf{N}^{n}$ is not squarefree, then for each $i, \Delta_{i, \alpha}$ is a cone and the cohomology of $C^{\bullet}(i, \alpha)$ is everywhere zero. It follows that the minimal resolution of $M$ is supported in squarefree degrees; see [BrHe95, Ya00].

Example 4.8. Let $A$ be the matrix of Example 3.2 and let $\alpha=(1,0,1,1)$. Then $l_{1}=l_{2}=0$,

$$
\begin{gathered}
C^{\bullet}(1, \alpha): \quad 0 \longrightarrow \mathbb{k} \longrightarrow \mathbb{k}^{2} \longrightarrow \mathbb{k} \longrightarrow 0 \\
C^{\bullet}(2, \alpha): \quad 0 \longrightarrow \mathbb{k} \longrightarrow \mathbb{k}^{2} \longrightarrow 0
\end{gathered}
$$

and

$$
C^{\bullet}(A, \alpha): \quad 0 \longrightarrow \mathbb{k}^{2} \longrightarrow \mathbb{k}^{4} \longrightarrow \mathbb{k} \longrightarrow 0 .
$$

It follows that $\operatorname{dim}_{\mathbb{k}} H^{0}\left(C^{\bullet}(A, \alpha)\right)=1$ and $b_{1, \alpha}\left(M_{A}\right)=1$, as expected. 


\section{LOCAL COHOMOLOGY OF $M$}

Let $A=\left(c_{i j} x^{\alpha_{i j}}\right)$ be an $s \times l$ multigraded squarefree matrix, $T$ be a squarefree solution of $E_{A}, \phi=\phi_{T}, M_{A}=$ Coker $\phi$ and $I_{1}, \ldots, I_{s}$ be the squarefree monomial ideals as in the previous section. We proceed with the notation and related remarks.

Notation 5.1.

- Let $F \subset[n]$. Let $H$ be any $R$-module. We write $H_{F}$ for the localization of $H$ at the powers of $x^{\underline{F}}$. In particular for $F=\left\{i_{1}, \ldots, i_{t}\right\}$, $R_{F}=\mathbb{k}\left[x_{1}, \ldots, x_{n}, x_{i_{1}}^{-1}, \ldots, x_{i_{t}}^{-1}\right]$. Let $u \in H$. We write $u_{F}$ to denote the image of $u$ in $H_{F}$ under the natural homomorphism $H \longrightarrow H_{F}$. If $\phi: H_{1} \longrightarrow H_{2}$ is an $R$-homomorphism we write $\phi_{F}:\left(H_{1}\right)_{F} \longrightarrow\left(H_{2}\right)_{F}$ for the induced homomorphism.

- We let $A_{F}=\left(c_{i j} x_{F}^{\alpha_{i j}}\right)$. We recall that $A$ is the matrix of $\phi: F_{1} \longrightarrow F_{0}$ with respect to bases $\left\{w_{i}: i=1, \ldots, l\right\}$ of $F_{1}$ and $\left\{v_{j}: j=1, \ldots, s\right\}$ of $F_{0}$. Thus $A_{F}$ is the matrix of $\phi_{F}:\left(F_{1}\right)_{F} \longrightarrow\left(F_{0}\right)_{F}$ with respect to the bases $\left\{\left(w_{i}\right)_{F}: i=1, \ldots, l\right\}$ of $\left(F_{1}\right)_{F}$ and $\left\{\left(v_{j}\right)_{F}: j=1, \ldots, s\right\}$ of $\left(F_{0}\right)_{F}$ and the multidegrees of $\left(w_{i}\right)_{F},\left(v_{j}\right)_{F}, i \in[l], j \in[s]$ are squarefree. For each $j$ we let $I_{j, F}=\left(I_{j}\right)_{F}$.

- Let $\Delta$ be a simplicial complex on $[n]$ and let $\alpha \in \mathbb{Z}^{n}$. We let

$$
\Delta^{\alpha}=\left\{\tau: \tau \cap \sigma_{a^{-}}=\emptyset, \tau \cup \sigma_{\alpha} \in \Delta\right\} .
$$

We note that if $\alpha=-\alpha^{-}$and $\sigma_{\alpha^{+}}=\emptyset$, then $\Delta^{\alpha}$ is by definition the link of $\sigma_{\alpha}$ in $\Delta$.

- Let $F, G \subset[n]$. Let $N$ be an $R$-module. We let $\theta_{F, G}: M_{F} \longrightarrow M_{G}$, $\theta_{F, G}\left(u_{F}\right)=u_{G}$ if $G=F h$ and zero otherwise. We let $K\left(x^{\infty}, N\right)$ be the complex

$$
K\left(x^{\infty}, N\right): 0 \rightarrow N \stackrel{\theta^{0}}{\longrightarrow} \bigoplus_{\substack{|F|=1 \\ F \subset[n]}} N_{F} \stackrel{\theta^{1}}{\rightarrow} \cdots \rightarrow N_{[n]} \rightarrow 0,
$$

where $\left.\theta^{r}\right|_{N_{F}}=\left(\theta_{F, G}\right)$. It is well known that for any multigraded module $N$ and $\alpha \in \mathbb{Z}^{n}$,

$$
H_{m}^{i}(N)_{\alpha}=H^{i}\left(K\left(x^{\infty}, N\right)_{\alpha}\right) ;
$$

see [BrHe98]. Moreover when $I$ is a squarefree monomial ideal, then by reordering the variables of $R$ so that the indices of $\sigma_{\alpha^{-}}$are at the end of $[n]$ one gets

$$
K\left(x^{\infty}, R / I\right)_{\alpha} \cong C^{\bullet}\left(\left(\Delta_{I}\right)^{\alpha}\right)\left[-\left|\sigma_{\alpha^{-}}\right|-1\right]
$$

and

$$
\operatorname{dim}_{\mathbb{k}} H_{m}^{i}(R / I)_{\alpha}=\operatorname{dim}_{\mathbb{k}} H^{i-\left|\sigma_{\alpha}-\right|-1}\left(C^{\bullet}\left(\left(\Delta_{I}\right)^{\alpha}\right)\right) ;
$$

see [St83] or [BrHe98].

- We recall from the previous section that if $\alpha \in \mathbf{Z}^{n}$, then $\alpha_{i}=\alpha-\beta_{i}$. We denote by $\Delta_{j}^{\alpha}$ the complex $\left(\Delta_{I_{j}}\right)^{\alpha_{j}}$. Thus

$$
\Delta_{j}^{\alpha}=\left\{\tau: \tau \cap \sigma_{a_{j-}}=\emptyset, \tau \cup \sigma_{\alpha_{j}} \in \Delta_{I_{j}}\right\} .
$$

If $F \subset[n]$ we let $B_{j, F}:=\left\{\overline{x^{\gamma} v_{j, F}}: x^{\gamma} \in R_{F}, x^{\gamma} \notin I_{j, F}\right\}$. We let

$$
B_{F}(A)=\bigcup_{j} B_{j, F}
$$


We note that $B_{j, F}=\left\{\overline{x^{\gamma} v_{j, F}}: \sigma_{\gamma^{-}} \subset F, F \cup \sigma_{\gamma^{+}} \in \Delta_{I_{j}}\right\}$. Moreover $\operatorname{deg}\left(\overline{x^{\gamma} v_{j, F}}\right)=$ $\gamma+\beta_{j}$. Thus the elements of $B_{F}(A)$ of degree $\alpha$ form the set $B_{F}(A)_{\alpha}=B_{F}(A) \cap$ $\left\{\overline{x^{\gamma} v_{i, F}}: \gamma+\beta_{i}=\alpha, i=1, \ldots, s\right\}=\left\{\overline{x^{\alpha_{i}} v_{i F}}: \sigma_{\alpha_{i}^{-}} \subset F, F \widehat{\sigma_{\alpha_{i}^{-}}} \in \Delta_{i}^{\alpha}, i=\right.$ $1, \ldots, s\}$. In the next theorem we determine a $\mathbb{k}$-basis for $K\left(x^{\infty}, M_{A}\right)_{\alpha}^{r}$ and its various homological components.

Theorem 5.3. Let $A$ be as above. Then

(1) $B_{F}(A)$ is a multigraded $\mathbb{k}$-basis for $\left(M_{A}\right)_{F}$. Moreover if $x^{\gamma} \in I_{i, F}$, then

$$
\overline{x^{\gamma} v_{i, F}}=\sum_{j<i} r_{i, j, \gamma+} \underline{\bar{F}} \overline{x^{\gamma+\beta_{i}-\beta_{j}} v_{j, F}} .
$$

(2) Let $\alpha \in \mathbb{Z}^{n}$. The set

$$
B_{\alpha}(A)=\bigcup_{F \subset[n]} B_{F}(A)_{\alpha}
$$

is a $\mathbb{k}$-basis for $K\left(x^{\infty}, M_{A}\right)_{\alpha}$.

(3) The set

$$
B_{\alpha, r}(A)=\left\{\overline{x^{\alpha_{i}} v_{i}}: F \subset[n],|F|=r, \sigma_{\alpha_{i}^{-}} \subset F, F \widehat{\sigma_{\alpha_{i}^{-}}} \in \Delta_{i}^{\alpha}\right\}
$$

is a $\mathbb{k}$-basis for $K\left(x^{\infty}, M_{A}\right)_{\alpha}^{r}$.

Proof. We prove the first claim. The rest follows by degree consideration. First we prove linear independence. Suppose that $x^{\gamma} \notin I_{j, F}$. Let $\beta \in \mathbb{N}^{n}$ such that $\sigma_{\gamma^{-}} \subset \sigma_{\beta} \subset F$. Then $x^{\beta} x^{\gamma} \notin I_{j}$. Thus by clearing denominators, any possible linear dependence relation on the elements of $B_{F}(A)$ corresponds to a linear dependence relation on the elements of $B\left(M_{A}\right)$.

Next we show that $B_{F}(A)$ spans $\left(M_{A}\right)_{F}$. Let $\gamma \in \mathbb{N}^{n}$ such that $x^{\gamma}$ is a generator of $I_{i, F}$. Then $x^{\gamma^{+}} x^{\underline{F}}$ is a generator of $I_{i}$. By Corollary 2.4 it follows that

$$
\overline{x^{\gamma^{+}+\underline{F}} v_{i}}=\sum_{j<i} r_{i, j, \gamma^{+}+\underline{F}} \overline{x^{\gamma^{+}+\beta_{i}-\beta_{j}} v_{j}}
$$

Localizing at the powers of $x^{\underline{F}}$ and dividing by $x^{\gamma^{-}} x^{\underline{F}}$ we get the desired claim.

Next we describe the complex that will be used to compute $H_{m}^{i}\left(M_{A}\right)_{\alpha}$. First we need to define one more sign: let $\sigma \subset F$. We reorder $F$ so that the elements of $\sigma$ are at the end of $F$. If the number of transpositions needed to do this is even, we let $t(\sigma, F):=1$; otherwise we let $t(\sigma, F):=-1$. If $h \notin F$, it is direct to verify that

$$
t(\sigma, F) \operatorname{sgn}[h, F h \hat{\sigma}]=t(\sigma, F h) \operatorname{sgn}[h, F h] .
$$

Theorem 5.4. Let $A$ be as above and $\alpha \in \mathbb{Z}^{n}$. Let $\left(L_{j}^{\alpha}\right)^{\bullet}=C^{\bullet}\left(\Delta_{j}^{a}\right)$ and $l_{j}^{-}=$ $\left|\sigma_{a_{i}^{-}}\right|-\left|\sigma_{a_{1}^{-}}\right|$. For each $r \in \mathbb{Z}$ we let

$$
\left(L^{\alpha}\right)^{r}:=\sum_{i=1}^{r} L_{i}^{r-l_{i}^{-}}
$$


and $d^{r}:\left(L^{\alpha}\right)^{r} \longrightarrow\left(L^{\alpha}\right)^{r+1}$ be such that when $\tau \in \Delta_{i}^{\alpha},|\tau|=r+1-l_{i}^{-}$, then

$$
\begin{aligned}
\tau^{*} & \mapsto \sum_{h \in V_{\tau, \Delta_{i}^{\alpha}, 1}} \operatorname{sgn}[h, \tau h](\tau h)^{*} \\
& +\sum_{h \in V_{\tau, \Delta_{i}^{\alpha}, 2}} \operatorname{sgn}[h, \tau h] \sum_{j<i} r_{i, j, \alpha_{i}^{-}+\underline{\tau h}} \frac{t\left(\sigma_{\alpha_{i}^{-}}, \tau h \sigma_{\alpha_{i}^{-}}\right)}{t\left(\sigma_{\alpha_{j}^{-}}, \tau h \sigma_{\alpha_{i}^{-}}\right)},\left(\tau h \sigma_{\alpha_{i}^{-}} \widehat{\sigma_{\alpha_{j}^{-}}}\right)^{*} .
\end{aligned}
$$

$\left(\left(L^{\alpha}\right)^{\bullet}, d^{\bullet}\right)$ is a cochain complex and

$$
\operatorname{dim}_{\mathbb{k}} H_{m}^{i}\left(M_{A}\right)_{\alpha}=\operatorname{dim}_{\mathbb{k}} H^{i-\left|\sigma_{\alpha_{1}^{-}}\right|-1}\left(\left(L^{\alpha}\right)^{\bullet}\right) .
$$

Proof. There is an isomorphism of vector spaces

$$
K\left(x^{\infty}, M_{A}\right)_{\alpha}^{r} \cong \sum_{i}\left(L_{i}^{\alpha}\right)^{r-\left|\sigma_{a_{i}}-\right|-1},
$$

where

$$
\overline{x^{\alpha_{i}} v_{i, F}} \mapsto\left(F \widehat{\sigma_{a_{i}^{-}}}\right)^{*} .
$$

It is routine to show that the following diagram commutes:

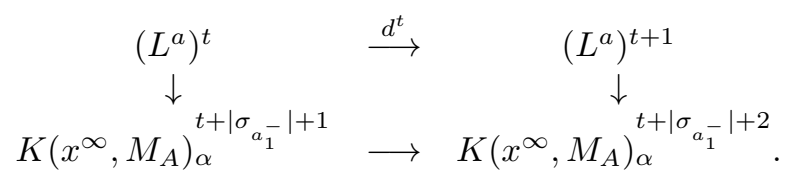

Example 5.5. Let $A$ be the matrix of Example 3.2 and let $\alpha=(0,-1,-1,0)$. Then $\alpha_{1}=(0,-1,-1,-1), \alpha_{2}=(-1,-1,-1,0), l_{1}^{-}=l_{2}^{-}=0$. Moreover $\Delta_{1}^{\alpha}=$ $\Delta_{2}^{\alpha}=\{\emptyset\}$,

$$
\left(L^{\alpha}\right)^{\bullet}: \quad 0 \longrightarrow \mathbb{k}^{2} \longrightarrow 0,
$$

and $\operatorname{dim}_{\mathbb{k}} H^{-1}\left(L^{\alpha}\right)=2$. It follows that $\operatorname{dim}_{\mathbb{k}} H_{m}^{3}\left(M_{A}\right)_{\alpha}=2$. We go into more detail for the case $\alpha=(0,0,0,0)$. Here $\alpha_{1}=(0,0,0,-1), \alpha_{2}=(-1,0,0,0), l_{1}^{-}=l_{2}^{-}=0$, $\sigma_{\alpha_{1}^{-}}=\{4\}$ and $\sigma_{\alpha_{2}^{-}}=\left\{1^{\prime}\right\} . \Delta_{1}^{\alpha}$ has as facets the boundary of the triangle $\{1,2,3\}$ while the facets of $\Delta_{2}^{\alpha}$ are $\{4\}$ and $\{2,3\}$. We have

$$
L^{\alpha}: 0 \longrightarrow \mathbb{k}^{2} \longrightarrow \mathbb{k}^{6} \longrightarrow \mathbb{k}^{4} \longrightarrow 0
$$

with zero cohomology at all homological degrees. For $\tau=\{2\} \in \Delta_{2}^{\alpha}, V_{\tau, \Delta_{2}^{\alpha}, 1}=\{3\}$, $V_{\tau, \Delta_{2}^{\alpha}, 2}=\{4\}$ and $d^{0}\left(\tau^{*}\right)=\tau_{1}-\tau_{2}$, where $\tau_{2}=\{2,3\}$ (in $\Delta_{2}^{\alpha}$ ) and $\tau_{1}=\{1,2\}$ (in $\left.\Delta_{1}^{\alpha}\right)$.

We finish this section with a corollary whose proof is immediate.

Corollary 5.6. Let $A$ be as above. If for some $\alpha \in \mathbb{Z}^{n}, H_{m}^{i}(M)_{\alpha} \neq 0$, then $\operatorname{dim}_{\mathbb{k}} H_{m}^{i}\left(M_{A}\right)_{\beta}=\operatorname{dim}_{\mathbb{k}} H_{m}^{i}\left(M_{A}\right)_{\alpha}$ for all $\beta \in \mathbb{Z}^{n}$ such that $\sigma_{\beta^{+}}=\sigma_{\alpha^{+}}$and $\sigma_{\beta^{-}}=$ $\sigma_{\alpha^{-}}$. 


\section{REFERENCES}

[BrHe95] W. Bruns And J. Herzog, On multigraded resolutions, Math. Proc. Camb. Phil. Soc., 118 (1995), 245-257. MR.1341789 (96g:13013)

[BrHe98] W. Bruns and J. Herzog, Cohen-Macaulay Rings, Cambridge University Press, 1998. MR:1251956 (95h:13020)

[Ch06] H. Charalambous, Multigraded Modules and Simplicial complexes, Proceedings of the 6th Panhellenic Conference in Algebra and Number Theory, Aristotle Univ. Thessalonike, 2006, 21-24.

[ChDe01] H. Charalambous and C. Deno, Multigraded modules, New York Journal of Mathematics, 7 (2001), 1-6. MR.1817761 (2002a:13009)

[ChTc03] H. Charalambous and A. TChernev, Free resolutions for multigraded modules: A generalization of Taylor's construction, Math. Res. Lett., 10 (2003), 535-550. MR $1995792(2004 \mathrm{e}: 13020)$

[Ei97] D. Eisenbud, Commutative Algebra with a View toward Algebraic Geometry, Springer Verlag, 1997. MR1322960 (97a:13001)

[Ho77] M. Hochster, Cohen-Macaulay rings, combinatorics, and simplicial complexes, Ring Theory. II, Lect. Notes in Pure and Applied Math., 26, M. Dekker, 1977, 171-223. MR0441987 (56:376)

[MiSt05] E. Miller AND B. Sturmfels, Combinatorial Commutative Algebra, Graduate Texts in Mathematics, 227, Springer Verlag, 2005. MR2110098 (2006d:13001)

[St83] R. Stanley, Combinatorics and Commutative Algebra, Birkhäuser, 1983. MR725505 (85b:05002)

[Ya00] K. YANAGAWA, Alexander duality for Stanley-Reisner rings and squarefree $\mathbf{N}^{n}$. graded modules. J. Algebra, 225 (2000), 630-645. MR.1741555 (2000m:13036)

Department of Mathematics, Aristotle University of Greece, Thessaloniki, 54124, GreECE

E-mail address: hara@math.auth.gr 